\title{
ON THE TOUGHNESS OF DUCTILE ADHESIVE JOINTS
}

\author{
VIGGO TVERGAARD \\ Department of Solid Mechanics, Technical University of Denmark, Lyngby, Denmark \\ and \\ JOHN W. HUTCHINSON \\ Division of Applied Sciences, Harvard University, Cambridge, Massachussetts, U.S.A.
}

\begin{abstract}
Crack propagation along one of the interfaces between a thin ductile adhesive layer and the elastic substrates it joins is considered. The layer is taken as being elastic-plastic, and the fracture process of the interface is modeled by a traction-separation law, characterized by the peak separation stress $\hat{\sigma}$ and the work of separation per unit area $\Gamma_{0}$. Crack growth resistance curves for mode I loading of the adhesive joint are computed, with emphasis on steady-state toughness, as a function of three extrinsic effects : layer thickness, layer-substrate modulus mismatch, and initial residual stress in the layer. Conditions under which separation first occurs well ahead of the initial crack tip are discussed.
\end{abstract}

\section{SPECIFICATION OF THE MODEL}

This paper continues the study begun by Tvergaard and Hutchinson (1994) in which an embedded fracture zone model is applied to the mode I fracture of an adhesive joint comprised of a thin elastic-plastic metal layer joining two elastic substrates. The present work employs the model to investigate the influence on joint toughness of both the elastic mismatch between the layer and the substrates and the residual stress in the layer. As in the earlier study, the thickness of the ductile layer is another extrinsic variable which comes into play.

The approach adopted was first introduced by Needleman (1987) to study particle debonding in metal matrices and subsequently by Tvergaard and Hutchinson (1992, 1993) to model crack growth resistance in homogeneous solids and along interfaces. A traction-separation law simulating the fracture process is embedded within an elastic-plastic continuum as a boundary condition along the line extending ahead of the crack. In the case of an interface joining dissimilar materials, the separation law necessarily involves both the normal and shear tractions and the two associated relative displacements of the surfaces across the interface. 

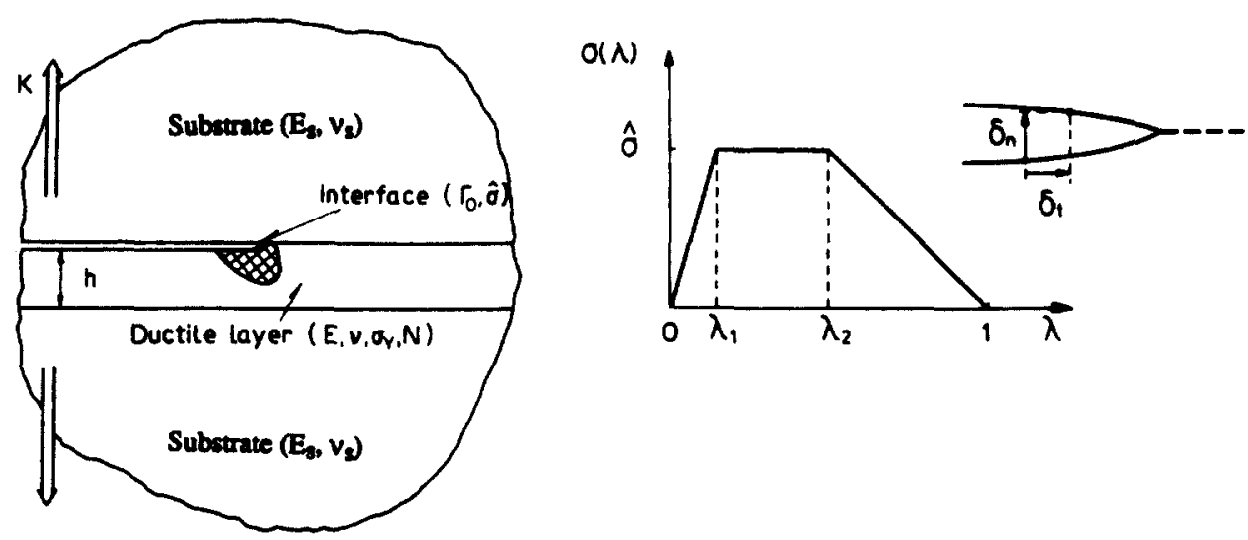

Fig. 1. Geometry of system and traction-separation relation for interface.

\subsection{Traction-separation law for the interface}

Following the notation for the law introduced in Tvergaard and Hutchinson (1993, 1994) let $\delta_{\mathrm{n}}$ and $\delta_{\mathrm{t}}$ be the normal and tangential components of the relative displacement of the crack faces across the interface in the zone where the fracture processes are occurring, as indicated in Fig. 1. Let $\delta_{\mathrm{n}}^{\mathrm{c}}$ and $\delta_{\mathrm{t}}^{\mathrm{c}}$ be critical values of these displacement components, and define a single nondimensional separation measure as

$$
\lambda=\sqrt{\left(\delta_{\mathrm{n}} / \delta_{\mathrm{n}}^{\mathrm{c}}\right)^{2}+\left(\delta_{\mathrm{t}} / \delta_{\mathrm{t}}^{\mathrm{c}}\right)^{2}}
$$

such that the tractions drop to zero when $\lambda=1$. With $\sigma(\lambda)$ displayed in Fig. 1, a potential from which the tractions are derived is defined as

$$
\Phi\left(\delta_{\mathrm{n}}, \delta_{\mathrm{t}}\right)=\delta_{\mathrm{n}}^{\mathrm{c}} \int_{0}^{\lambda} \sigma\left(\lambda^{\prime}\right) \mathrm{d} \lambda^{\prime} .
$$

The normal and tangential components of the traction acting on the interface in the fracture process zone are given by

$$
T_{\mathrm{n}}=\frac{\partial \Phi}{\partial \delta_{\mathrm{n}}}=\frac{\sigma(\lambda)}{\lambda} \frac{\delta_{\mathrm{n}}}{\delta_{\mathrm{n}}^{\mathrm{c}}}, \quad T_{\mathrm{t}}=\frac{\partial \Phi}{\partial \delta_{\mathrm{t}}}=\frac{\sigma(\lambda)}{\lambda} \frac{\delta_{\mathrm{t}}}{\delta_{\mathrm{t}}^{\mathrm{c}}} \frac{\delta_{\mathrm{n}}^{\mathrm{c}}}{\delta_{\mathrm{t}}^{\mathrm{c}}} .
$$

The traction law under a purely normal separation $\left(\delta_{\mathrm{t}}=0\right)$ is $T_{\mathrm{n}}=\sigma(\lambda)$ where $\lambda=\delta_{\mathrm{n}} / \delta_{\mathrm{n}}^{\mathrm{c}}$. Under a purely tangential displacement $\left(\delta_{\mathrm{n}}=0\right), T_{\mathrm{t}}=\left(\delta_{\mathrm{n}}^{\mathrm{c}} / \delta_{\mathrm{t}}^{\mathrm{c}}\right) \sigma(\lambda)$ where $\lambda=\delta_{\mathrm{t}} / \delta_{\mathrm{t}}^{\mathrm{c}}$. The peak normal traction under pure normal separation is $\hat{\sigma}$, and the peak shear traction is $\left(\delta_{\mathrm{n}}^{\mathrm{c}} / \delta_{\mathrm{t}}^{\mathrm{c}}\right) \hat{\sigma}$ in a pure tangential "separation". The work of separation per unit area of interface is given by (1.2) with $\lambda=1$. For the separation function $\sigma(\lambda)$ specified in Fig. 1,

$$
\Gamma_{0}=\frac{1}{2} \hat{\sigma} \delta_{\mathrm{n}}^{\mathrm{c}}\left[1-\lambda_{1}+\lambda_{2}\right] .
$$

The parameters governing the separation law of the interface are the work of the fracture process $\Gamma_{0}$, the peak stress quantity $\hat{\sigma}$, and the critical displacement ratio $\delta_{\mathrm{n}}^{\mathrm{c}} / \delta_{\mathrm{t}}^{\mathrm{c}}$, together with the factors $\lambda_{1}$ and $\lambda_{2}$ governing the shape of the separation 
function. Note that use of the potential ensures that the work of separation is $\Gamma_{0}$ regardless of the combination of normal and tangential displacements taking place in the process zone. Experience gained in the earlier studies suggests that the details of the shape of the separation law are relatively unimportant. The loading in the present ductile layer problem is mode I. Nevertheless, the asymmetry introduced by the existence of the crack on one interface and not the other gives rise to some tangential separation. However, the separation displacements are predominantly normal, such that the choice of the ratio $\delta_{\mathrm{n}}^{\mathrm{c}} / \delta_{\mathrm{t}}^{\mathrm{c}}$ also has relatively little influence on the predictions of the macroscopic toughness. The two most important parameters characterizing the fracture process in this model are $\Gamma_{0}$ and $\hat{\sigma}$.

\subsection{Continuum properties of the ductile adhesive and the elastic substrates}

The layer has thickness $h$ and is assumed to be elastically isotropic with Young's modulus $E$ and Poisson's ratio $v$. The residual stress state in the unloaded layer is assumed to be equibiaxial, acting parallel to the plane of the layer, of magnitude $\sigma_{\mathrm{R}}$. Commonly, when a metal layer joins two ceramic substrates, a residual tensile stress in the thin metal layer develops due to thermal expansion mismatch and the consequence of cooling from a high bonding temperature. The plastic response of the layer material is characterized by $J_{2}$ flow theory, i.e. the standard isotropic hardening incremental plasticity theory based on the Mises invariant. The tensile curve of true stress versus true strain for the layer material is taken to be

$$
\begin{array}{lll}
\varepsilon=\sigma / E & \text { for } & \sigma \leqslant \sigma_{\mathrm{Y}} \\
\varepsilon=\left(\sigma_{\mathrm{Y}} / E\right)\left(\sigma / \sigma_{\mathrm{Y}}\right)^{1 / N} & \text { for } & \sigma>\sigma_{\mathrm{Y}}
\end{array}
$$

where $\sigma_{\mathrm{Y}}$ is the tensile yield stress and $N$ is the strain hardening exponent. The primary effect of the residual stress is its influence on the onset of yield when the joint is loaded.

The two elastic substrates joined by the adhesive layer are assumed to be identical and isotropic with Young's modulus $E_{\mathrm{s}}$ and Poisson's ratio $v_{\mathrm{s}}$.

\subsection{Mode I loading for long cracks and definition of steady-state toughness}

The focus in this paper is on symmetrically loaded joints containing interface cracks that are long compared to the extent of the plastic zones induced in the layer. Under this condition, the asymptotic problem indicated in Fig. 1 applies, wherein the crack is taken to be semi-infinite and is loaded remotely by the symmetric mode I stress field with amplitude given by the stress intensity factor $K$. Irwin's relation between the energy release rate $G$ and the stress intensity factor $K$ for a mode I, plane strain crack in an elastic solid is

$$
G=\frac{\left(1-v_{\mathrm{s}}^{2}\right)}{E_{\mathrm{s}}} K^{2} .
$$

This relation applies to the asymptotic problem of Fig. 1 with $G$ interpreted as the remote, or applied, energy release rate. The crack growth resistance $\Gamma$ is identified with $G$ under conditions of crack advance. There is one material length quantity, $R_{0}$, 
in the model, which was introduced in the study of mixed mode interface fracture by Tvergaard and Hutchinson (1993)

$$
R_{0}=\frac{2}{3 \pi\left(1-\beta^{2}\right)}\left[\frac{\left(1-v^{2}\right)}{E}+\frac{\left(1-v_{\mathrm{s}}^{2}\right)}{E_{\mathrm{s}}}\right]^{-1} \frac{\Gamma_{0}}{\sigma_{\mathrm{Y}}^{2}}
$$

where $\beta$ is the second Dundurs elastic mismatch parameter

$$
\beta=\frac{1}{2} \frac{\mu\left(1-2 v_{\mathrm{s}}\right)-\mu_{\mathrm{s}}(1-2 v)}{\mu\left(1-v_{s}\right)+\mu_{\mathrm{s}}(1-v)}
$$

and $\mu$ and $\mu_{\mathrm{s}}$ are the shear moduli. In the absence of elastic mismatch (1.7) reduces to

$$
R_{0}=\frac{1}{3 \pi\left(1-v^{2}\right)} \frac{E \Gamma_{0}}{\sigma_{\mathrm{Y}}^{2}} .
$$

The length quantity $R_{0}$ can be thought of as an estimate of the size of the plastic zone of the interface crack when $\Gamma=\Gamma_{0}$ and $h \gg R_{0}$.

The model has been used to compute the history of $\Gamma$ as a function of crack advance $\Delta a$ as dependent on the parameters of the system. From this resistance curve data, one can identify a toughness level characterizing initiation of crack growth and an asymptote, denoted by $\Gamma_{\mathrm{ss}}$, characterizing the steady-state condition wherein the crack advances under constant $\Gamma$. Typically, this asymptote is attained after a crack advance on the order of several times $R_{0}$. The steady-state toughness, $\Gamma_{\mathrm{ss}}$, provides the most meaningful measure of the joint toughness, and it will be this quantity which will be featured in the sequel. The nondimensional relation between the steady-state toughness and the parameters of the model is

$$
\frac{\Gamma_{\mathrm{ss}}}{\Gamma_{0}}=F\left(\frac{\hat{\sigma}}{\sigma_{\mathrm{Y}}}, N, \frac{h}{R_{0}}, \frac{E_{\mathrm{s}}}{E}, \frac{\sigma_{\mathrm{R}}}{\sigma_{\mathrm{Y}}}\right) .
$$

There is also a weak dependence on $\delta_{\mathrm{t}}^{\mathrm{c}} / \delta_{\mathrm{n}}^{\mathrm{c}}, v, v_{\mathrm{S}}, \sigma_{\mathrm{Y}} / E, \lambda_{1}$ and $\lambda_{2}$. Given the large number of parameters in the model, it is not possible to present or even compute the full parametric dependence. In this paper, attention is directed to the parameters shown explicitly in (1.10), with the exception of $N$, which is fixed at 0.1 in all the computations corresponding to a typical strain hardening level. Each value of $\Gamma_{\mathrm{ss}} / \Gamma_{0}$ is obtained by computing the full resistance curve, starting from initiation to a level of a crack advance, $\Delta a$, such that $\Gamma$ approximately attains the asymptote, and, consequently, extensive numerical computation lies behind the results reported in Section 3.

\section{NUMERICAL METHOD}

The problem considered is an extension of the problem analyzed by Tvergaard and Hutchinson (1994), and the numerical method employed is essentially the same as that presented in that earlier paper. The method exploits the fact that the plastic 
deformation takes place only in the thin layer of thickness $h$, while the solids on either side of the layer remain elastic. The region analyzed numerically is divided into three sub-regions, where the outer regions comprising the elastic substrates are semi-circular with radius $A_{0}$, and region occupied by the layer is rectangular with dimensions $h$ by $2 A_{0}$. The finite element mesh consists of quadrilaterals, each built up of four triangular, linear-displacement elements. The meshes used for the substrate region are identical to those employed in the earlier reference. In the layer the quadrilaterals are rectangular with edge nodes located so that they fit the edge nodes of the adjacent region. The initial crack tip is located at $x_{1}=x_{2}=0$, and a uniform mesh region of length $B_{0}$ is used in front of the initial crack tip to model crack growth. The length of one square element in this uniformly meshed region is denoted by $\Delta_{0}$.

On the circular edges of the substrate regions loads corresponding to the tractions of the mode I stress field are applied, with amplitude $K$. At the two ends of the thin layer the edge loads are neglected, which introduces a very small error as long as $h / A_{0}$ is small. At the interface between the lower substrate and the layer, the conditions to be satisfied are

$$
\begin{array}{cl}
u^{1}\left(\eta_{+}\right)=u^{1}\left(\eta_{-}\right), & u^{2}\left(\eta_{+}\right)=u^{2}\left(\eta_{-}\right) \\
T^{1}\left(\eta_{+}\right)=-T^{1}\left(\eta_{-}\right), & T^{2}\left(\eta_{+}\right)=-T^{2}\left(\eta_{-}\right)
\end{array}
$$

where $\eta$ is the coordinate along the interface and the " + " denotes a value just above the interface and the "- " denotes a value just below. Along the upper interface, the initial crack surfaces for $x_{1}<0$ are traction-free, while for $x_{1}>0$ the displacements and tractions are related by the traction separation law of the interface of Section 1.1. Thus, for $x_{1}>0$

$$
\begin{array}{cl}
u^{1}\left(x_{1+}\right)-u^{1}\left(x_{1-}\right)=\delta_{1}\left(x_{1}\right), & u^{2}\left(x_{1+}\right)-u^{2}\left(x_{1-}\right)=\delta_{\mathrm{n}}\left(x_{1}\right) \\
T^{1}\left(x_{1+}\right)=-T^{1}\left(x_{1-}\right)=T_{\mathrm{t}}\left(x_{1}\right), & T^{2}\left(x_{1+}\right)=-T^{2}\left(x_{1-}\right)=T_{\mathrm{n}}\left(x_{1}\right) .
\end{array}
$$

For the upper substrate region, the linear elastic equations are solved once at the start to obtain linear relations between the nodal displacements along $x_{1}>0$, the corresponding nodal forces, and the load amplitude $K$, using a Rayleigh-Ritz finite element method. Similar linear relations are obtained between nodal displacements on the lower interface boundary, the corresponding nodal forces, and the amplitude $K$. In the layer, elastic-plastic deformations take place following a finite strain generalization of $J_{2}$ flow theory, as has been described in more detail in Tvergaard and Hutchinson (1992). Thus, solutions in the layer have to be obtained incrementally, and in each increment linear relations are obtained between nodal displacement increments and the corresponding nodal force increments on the upper and lower edges of the layer.

The relations for the three sub-regions are finally assembled using $(2.1)-(2.4)$ to obtain a set of linear algebraic equations for the increment in the load amplitude $K$ and the nodal displacement increments along the layer edges. On the initial part of the resistance curve, an increment in $K$ is prescribed, but this procedure is unstable when $K$ approaches its asymptote. Then, the Rayleigh-Ritz method is used to control a monotonic increase of displacement differences at the crack tip. 
In all of the computations, the properties of the elastic-plastic layer are specified by the parameters $\sigma_{\mathrm{Y}} / E=0.003, v=\frac{1}{3}$, and $N=0.1$. Different levels of elastic modulus mismatch between the layer and the substrates are considered $\left(E_{\mathrm{s}} / E=1,3,6\right.$ and 12), all with $v_{\mathrm{s}}=v=\frac{1}{3}$. The separation law is specified using $\delta_{\mathrm{n}}^{\mathrm{c}} / \delta_{\mathrm{t}}^{\mathrm{c}}=1, \lambda_{1}=0.15$ and $\lambda_{2}=0.5$ with $\hat{\sigma} / \sigma_{\mathrm{Y}}$ varied. The preceding paper established that the predictions for the present class of problems are not strongly sensitive to $\delta_{\mathrm{n}}^{\mathrm{c}} / \delta_{\mathrm{t}}^{\mathrm{c}}, \lambda_{1}$ or $\lambda_{2}$.

Let $\Delta_{0}$ denote the length of one of the small elements in the uniform mesh along the interface in the crack growth region. Most of the computations have been carried out for a region size specified by $A_{0}=44,000 \Delta_{0}$ and $B_{0}=40 \Delta_{0}$. Mesh refinements have been carried out to test convergence. Based on these trials the computations for the elastic mismatches with $E_{\mathrm{s}} / E=3,6$ and 12 were performed with $\Delta_{0}=10 \delta_{\mathrm{n}}^{\mathrm{c}}$, whereas the computations with no modulus mismatch were computed with $\Delta_{0}=5$ $\delta_{\mathrm{n}}^{\mathrm{c}}$. For the larger values of the layer thickness $h$, compared to $\Delta_{0}$, the mesh size is stretched across the layer in such a way that the row of quadrilateral elements along the crack plane are square. In each of the semi-circular elastic regions the number of triangular elements is 6248 and the number of nodal points is 3203 . In the thin elasticplastic layer the number of triangular elements is 8800 and the number of nodal points is 4531 .

\section{STEADY-STATE TOUGHNESS}

The full set of numerical results for $\Gamma_{\mathrm{ss}} / \Gamma_{0}$ as a function of $\hat{\sigma} / \sigma_{\mathrm{Y}}, h / R_{0}, E_{\mathrm{s}} / E$ and $\sigma_{\mathrm{R}} / \sigma_{\mathrm{Y}}$ are shown in the four parts of Fig. 2. Parts (a)-(d) correspond to values of $E_{\mathrm{s}} / E$ of $1,3,6$, and 12 , respectively. Figure 2 (a) and (c) display results for $\sigma_{\mathrm{R}}=0$ (the solid-line curves), as well as for a biaxial tensile residual stress in the layer nearly at yield, $\sigma_{\mathrm{R}} / \sigma_{0}=0.95$ (the dashed-line curves). The solid-line curves for the case of no elastic mismatch in Fig. 2(a) were presented earlier by Tvergaard and Hutchinson (1994).

The main qualitative trends evident in Fig. 2 can be summarized as follows. (i) Normalized steady-state toughness increases strongly with $\hat{\sigma} / \sigma_{\mathrm{Y}}$, with all other parameters held fixed. This dependence has been discussed in some detail in the authors' earlier papers on the model. (ii) Very thin layers, in the sense that $h / R_{0} \ll 1$, have almost no enhancement of toughness due to plastic deformation, i.e. $\Gamma_{\mathrm{ss}} \cong \Gamma_{0}$. This limit, in which the layer thickness is small compared to the size of the unconstraincd plastic zone, will be discussed further in Section 4. (iii) At the other limit, when the layer thickness is larger than the plastic zone $\left(h / R_{0} \gg 1\right), \Gamma_{\mathrm{ss}} / \Gamma_{0}$ becomes independent of $h$, because the interaction of the plastic zone with substrate on the other side of the uncracked interface becomes negligible. The value of $h / R_{0}$ at which the toughness hecomes independent of the layer thickness is a function of $\hat{\sigma} / \sigma_{\mathrm{Y}}$ because the plastic zone size increases with this parameter. (iv) The ratio of the substrate elastic modulus to that of the layer, $E_{\mathrm{s}} / E$, has a fairly significant effect such that joints with relatively stiff substrates have higher toughnesses, all other parameters remaining the same. (v) The residual stress in the layer $\sigma_{\mathrm{R}}$ has a major effect on joint toughness. Tensile residual stresses lower the joint toughness, while compressive stresses raise the toughness.

The influence of $E_{\mathrm{s}} / E$ on the normalized steady-state toughness is isolated in Fig. 


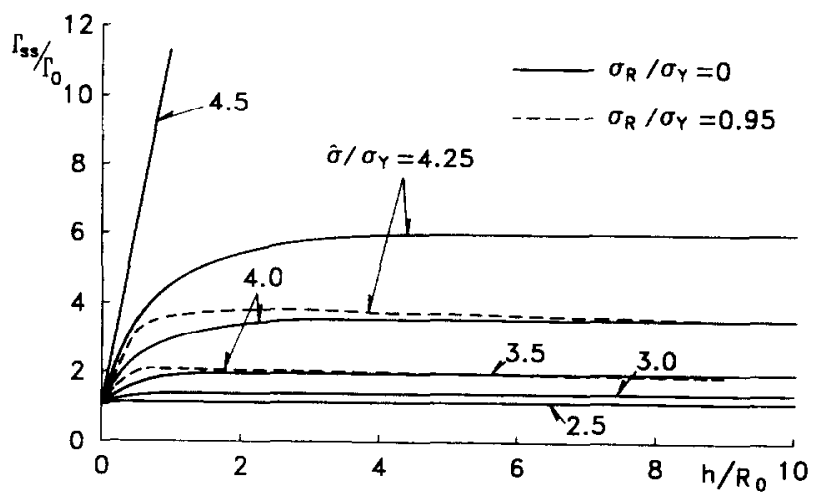

a)

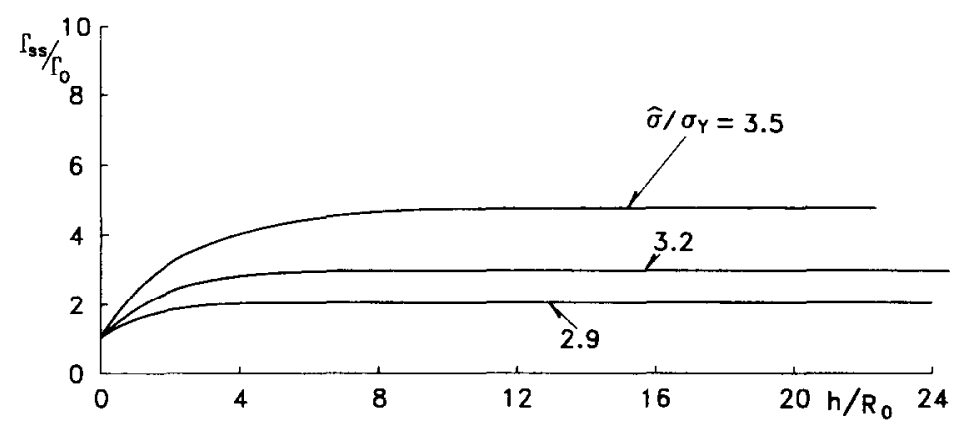

b)

Fig. 2. Normalized steady-state toughness of the joint as a function of layer thickness for various values of $\hat{\sigma} / \sigma_{\mathrm{Y}}$. (a) $E_{\mathrm{s}} / E=1$, (b) $E_{\mathrm{s}} / E=3$, (c) $E_{\mathrm{s}} / E=6$, and (d) $E_{\mathrm{s}} / E=12$. The other parameters are specified in the text.

3, where curves of $\Gamma_{\mathrm{ss}} / \Gamma_{0}$ as a function of $\hat{\sigma} / \sigma_{\mathrm{Y}}$ are plotted for large values of $h / R_{0}$ chosen to ensure that the toughness is independent of $h$. In the range of $\partial / \sigma_{\mathrm{Y}}$ for which the toughness is magnified above $\Gamma_{0}$, an increase in $E_{\mathrm{s}} / E$ leads to an increase in $\Gamma_{\mathrm{ss}} / \Gamma_{0}$. Judging from the results in Fig. 3, it appears that the full effect is achieved for modulus mismatches satisfying $E_{\mathrm{s}} / E>10$. It seems likely that the toughness enhancement is related to the ability of stiffer substrates to better shield the layer near the tip, thereby lowering the peak normal stress on the interface ahead of the tip.

A cross-plot of $\Gamma_{\mathrm{ss}} / \Gamma_{0}$ as a function of $\sigma_{\mathrm{R}} / \sigma_{\mathrm{Y}}$ is shown for one set of parameters in Fig. 4. The case shown has $E_{\mathrm{s}} / E=6, \hat{\sigma} / \sigma_{\mathrm{Y}}=3$, with $\sigma_{\mathrm{Y}} / E=0.003$ and $N=0.1$. The calculations were carried out using $h / R_{0}=13.46$, and thus these results are also in the range where the plastic zone does not extend across the layer and the joint toughness is independent of $h$. The role of residual stress is clearly significant. Qualitatively, the residual stress effect can be thought of as raising (when $\sigma_{\mathrm{R}}>0$ ) or lowering (when 


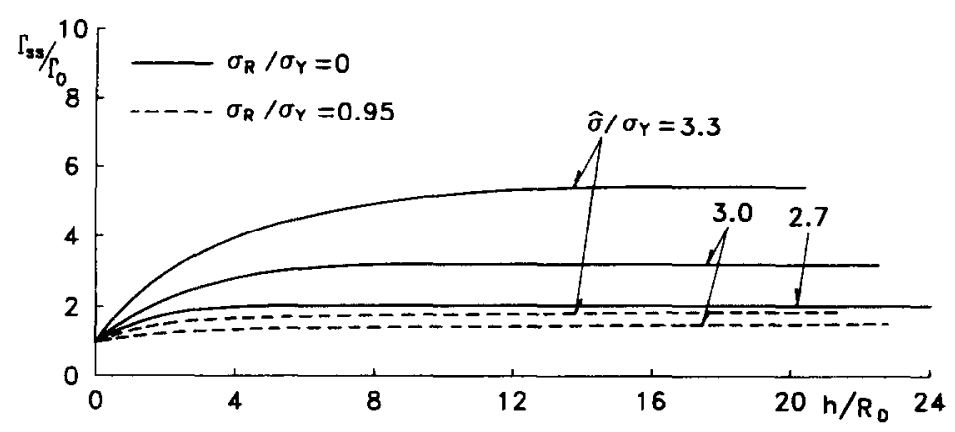

c)

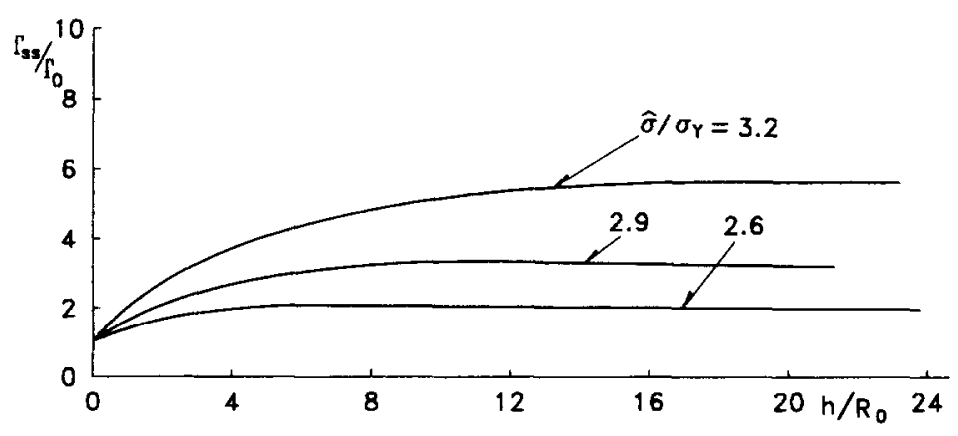

d)

Fig. 2. Continued.

$\sigma_{\mathrm{R}}<0$ ) the additional effective stress needed to cause yield, thereby suppressing or enhancing plastic deformation. To understand this, note that, with respect to plastic yielding, a residual biaxial stress $\sigma_{\mathrm{R}}$ acting parallel to the plane of the layer is equivalent to a uniaxial stress $-\sigma_{\mathrm{R}}$ acting normal to the plane of the layer. (This follows from superposition of a hydrostatic stress state $-\sigma_{\mathrm{R}} \delta_{i j}$, which has no effect on the yield condition.) Thus, the onset of plastic yielding at any point in the layer near the tip will be delayed (or advanced) to a higher (or lower) local stress normal to the interface resulting from the applied $K$, depending on whether $\sigma_{\mathrm{R}}>0$ (or $\left.\sigma_{\mathrm{R}}<0\right)$. The effect is roughly equivalent to decreasing or increasing $\hat{\sigma}$.

In the examples shown in Figs 2-4, the peak interface stress $\hat{\sigma}$ is attained immediately ahead of the crack tip such that debonding links back to the crack tip as crack advance occurs. The stress distribution in a thin metal layer ahead of the crack tip in the layersubstrate geometry considered here has also been studied by Varias et al. (1991) for the stationary crack without any debonding. The constraint imposed on the layer by the elastic substrates gives rise to a large component of hydrostatic tension in the 


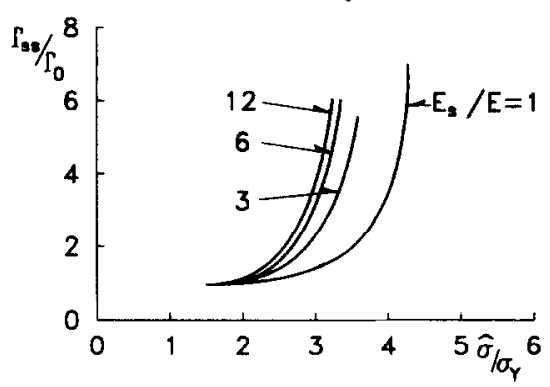

Fig. 3. Dependence of steady-state toughness of the joint on $\hat{\sigma} / \sigma_{\mathrm{Y}}$ for various $E_{\mathrm{s}} / E$, all for the limit of large $h / R_{0}$ for which the layer thickness exceeds the height of the plastic zone.

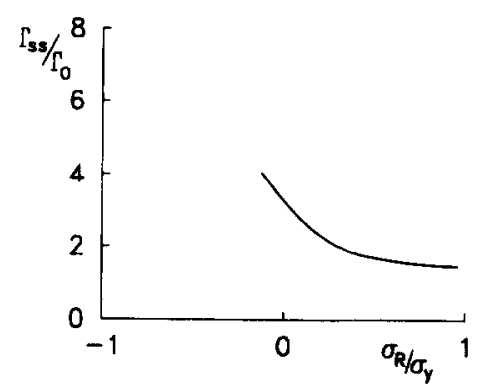

Fig. 4. Effect of residual stress on the steady-state toughness for the limit in which $h / R_{0} \gg 1$. In this example, $\hat{\sigma} / \sigma_{Y}=3$ and $E_{\mathrm{s}} / E=6$.

layer such that the peak in the interface stress occurs at a distance ahead of the tip. This is the location where the interface stress first attains $\hat{\sigma}$. If $\hat{\sigma} / \sigma_{\mathrm{Y}}$ is sufficiently large and if the layer is sufficiently thin, then the peak stress on the interface is attained so far ahead of the tip that debonded patches occur which are unconnected to the crack tip. An example for which this occurs is shown in Fig. 5 for the case where $\hat{\sigma} / \sigma_{\mathrm{Y}}=5$, $E_{\mathrm{s}} / E=1$ and $h / R_{0}=0.186$. The figure displays the deformed mesh and crack opening profile at three levels of applied load corresponding to $\Gamma / \Gamma_{0}=2.06,2.90$, and 3.73. At the lowest of the three load levels, the peak stress has just been attained and debonding of the interface has begun at a distance of approximately $2 h$ ahead of the blunted crack tip. At the two higher load levels, an isolated debonded patch opens and expands. Of course, the present calculations are two dimensional, while the actual process will be three dimensional. Debonding in roughly equiaxed patches well ahead of the crack tip has been observed and documented for $\mathrm{Au} / \mathrm{Al}_{2} \mathrm{O}_{3}$ interfaces by Reimanis et al. (1991) and Turner and Evans (1996) and has been studied theoretically by He et al. (1996).

\section{APPROXIMATE ANALYSIS OF MODEL FOR LARGE $\hat{\sigma} / \sigma_{\mathrm{Y}}$}

In this section an approximate analysis of the model will be carried out under the assumption that $\hat{\sigma} / \sigma_{\mathrm{Y}}$ is sufficiently large that interface debonding starts at a point $\hat{x}$ at least several layer thicknesses ahead of the tip. Thus, the focus will be on initiation 


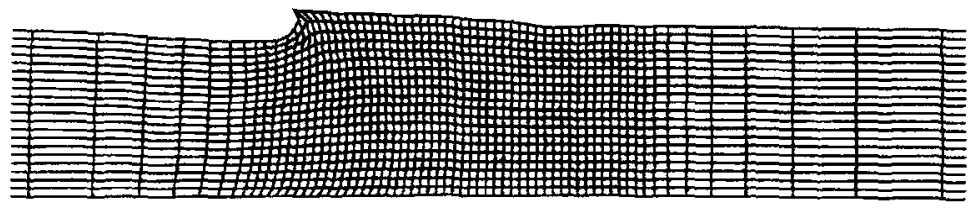

$\Gamma \Gamma_{0}=2.06$

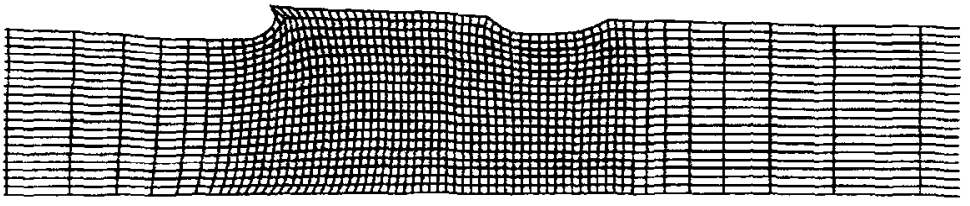

$\Gamma / \Gamma_{0}=2.90$

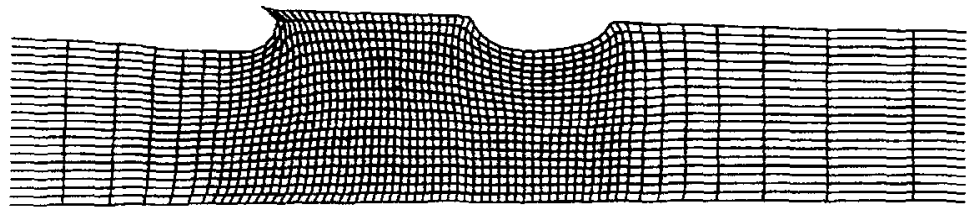

\section{$\Gamma / \Gamma 0=3.73$}

Fig. 5. An example illustrating debonding of a patch unconnected to the crack tip at three levels of load. For this example, $\hat{\sigma} / \sigma_{\mathrm{Y}}=5, E_{\mathrm{s}} / E=1$ and $h / R_{0}=0.186$.

of debonding in the regime discussed above where debonding starts in the form of isolated patches ahead of the tip. While highly approximate, the analysis does capture the qualitative dependencies on some of the most important parameters and informs aspects of behavior in the regime where debonding extends back to the tip.

Assume there is no residual stress in the layer and the layer material is elasticperfectly plastic. When the layer is very thin, the elastic stress distribution for the limit of zero layer thickness will be used to estimate the stress acting on the interface at the point where the peak stress is attained. That is, from $\sigma_{22}=K / \sqrt{2 \pi x_{1}}$, it follows that if $\hat{K}$ is the value of the stress intensity factor at which debonding starts

$$
\hat{\sigma}=\hat{K} / \sqrt{2 \pi \hat{x}} .
$$

In the portion of the layer between the tip and the point of initial debonding, i.e. $0<x_{1}<\hat{x}$, the $x_{1}$ component of stress increases linearly according to $\mathrm{d} \sigma_{11} / \mathrm{d} x_{1}=$ $(2 / \sqrt{3}) \sigma_{\mathrm{Y}} / h$. This follows from simple equilibrium and the fact that the layer undergoes plastic shearing along its top and bottom surfaces (Hill, 1950; Varias et al., 1991). The well-known stress state at the tip of a crack in an elastic-perfectly plastic material applies such that, at $x_{1}=0, \sigma_{11} \cong 2 \sigma_{\mathrm{Y}}$. Therefore, at the location of the peak stress, 
$\sigma_{11} \cong 2 \sigma_{\mathrm{Y}}+(2 / \sqrt{3}) \sigma_{\mathrm{Y}}(\hat{x} / h)$. The state of stress at the location of the peak satisfies yield and is approximately a state of hydrostatic tension superimposed on a state of uniaxial tension, with $\sigma_{22} \cong \sigma_{11}+\sigma_{\mathrm{Y}}$. Thus, a second approximate relation between the peak debonding stress and the location of the onset of debonding is

$$
\hat{\sigma} \cong 3 \sigma_{\mathrm{Y}}+(2 / \sqrt{3}) \sigma_{\mathrm{Y}}(\hat{x} / h) .
$$

Eliminate $\hat{x}$ from (4.1) and (4.2), using (1.6), to obtain the estimate of $\hat{\Gamma}$ at which the debonding condition is first attained

$$
\hat{\Gamma}=\sqrt{3} \pi\left(1-v_{\mathrm{s}}^{2}\right)\left(\frac{\hat{\sigma}}{\sigma_{\mathrm{Y}}}\right)^{2}\left(\frac{\hat{\sigma}}{\sigma_{\mathrm{Y}}}-3\right)_{E_{\mathrm{s}}}^{h \sigma_{\mathrm{Y}}^{2}} .
$$

The location of the point of first debond is

$$
\frac{\hat{x}}{h}=\frac{\sqrt{3}}{2}\left(\frac{\hat{\sigma}}{\sigma_{\mathrm{Y}}}-3\right)
$$

Note that $\hat{\Gamma}$ does not depend on $\Gamma_{0}$; it is associated with the attainment of a critical stress at some point on the interface ahead of the crack tip. Application of the above formulas is limited to values of $\hat{\sigma} / \sigma_{\mathrm{Y}}$ sufficiently large such that $\hat{x} / h \geqslant 2$, i.e. roughly $\hat{\sigma} / \sigma_{\mathrm{Y}} \geqslant 5$. Finally, normalize the estimate in (4.3) by $\Gamma_{0}$, using (1.7), to obtain

$$
\frac{\hat{\Gamma}}{\Gamma_{0}}=\frac{\left(1-v_{\mathrm{s}}^{2}\right)}{\sqrt{3}}\left(\frac{\hat{\sigma}}{\sigma_{\mathrm{Y}}}\right)^{2}\left(\frac{\hat{\sigma}}{\sigma_{\mathrm{Y}}}-3\right) \frac{E^{*}}{E_{\mathrm{s}}} \frac{h}{R_{0}}
$$

where

$$
\frac{1}{E^{*}}=\frac{\left(1-\beta^{2}\right)}{2}\left(\frac{1-v^{2}}{E}+\frac{1-v_{\mathrm{s}}^{2}}{E_{\mathrm{s}}}\right)
$$

Formulas (4.3) and (4.5) reveal the exceptionally strong dependence on $\hat{\sigma} / \sigma_{\mathrm{Y}}$, which is also evident in the other regime in the plots of steady-state toughness in Fig. 2. Note that the trend of $\widehat{\Gamma} / \Gamma_{0}$ with $E_{\mathrm{s}} / E$ implied by (4.5) is opposite to that for $\Gamma_{\mathrm{ss}} / \Gamma_{0}$ in Fig. 3 in the range $h / R_{0} \gg 1$. Numerical calculations for $\hat{\Gamma} / \Gamma_{0}$ as a function of $E_{\mathrm{s}} / E$ in the range of small $h / R_{0}$ with large $\hat{\sigma} / \sigma_{\mathrm{Y}}$ have not been carried out.

Finally, a connection is noted between the approximate results for the onset of debonding and the numerical results for steady-state toughness in Fig. 2. From the plots in Fig. 2, it can be seen that in the range of small $h / R_{0}\left(\right.$ e.g. $\left.h / R_{0}<1 / 2\right)$ the normalized joint toughness varies approximately linearly with layer thickness according to

$$
\frac{\Gamma_{\mathrm{SS}}}{\Gamma_{0}} \cong 1+C \frac{h}{R_{0}}
$$

or

$$
\Gamma_{\mathrm{SS}} \cong \Gamma_{0}+C \frac{h \Gamma_{0}}{R_{0}}=\Gamma_{0}+3 \pi C \frac{h \sigma_{\mathrm{Y}}^{2}}{E^{*}}
$$


where $C$ depends on $\hat{\sigma} / \sigma_{\mathrm{Y}}$ and $N$ but not on $\Gamma_{0}$. In this range, the enhancement of joint toughness above the work of separation of the interface is independent of $\Gamma_{0}$, as is the case for the result in (4.3). In fact, it can be noted that the enhancement in steady-state toughness in (4.8) has precisely the same parametric dependence as (4.3). Qualitatively, the trend of $\hat{\Gamma}$ with $\hat{\sigma}$ given by (4.3) reflects the trends shown in Fig. 2 in the range of small $h / R_{0}$.

\section{ACKNOWLEDGEMENT}

The work of JWH was supported in part by the National Science Foundation under Grants MSS-92-02141 and DMR-94-00396 and by the Division of Applied Sciences, Harvard University.

\section{REFERENCES}

He, M. Y., Evans, A. G. and Hutchinson, J. W. (1996) Interface cracking phenomena in constrained metal layers. Acta Metall. Mater., in press.

Hill, R. (1950) The Mathematical Theory of Plasticity. Clarendon Press, Oxford.

Needleman, A. (1987) A continuum model for void nucleation by inclusion debonding. J. Appl. Mech. 54, 525-531.

Reimanis, I. E., Dalgleish, B. I. and Evans, A. G. (1991) The fracture resistance of a model metal/ceramic interface. Acta Metall. Mater. 39, 3133-3141.

Turner, M. R. and Evans, A. G. (1996) An experimental study of the mechanisms of crack extension along an oxide/metal interface. Acta Metall. Mater., in press.

Tvergaard, V. and Hutchinson, J. W. (1992) The relation between crack growth resistance and fracture process parameters in elastic-plastic solids. J. Mech. Phys. Solids 40, 1377-1397.

Tvergaard, V. and Hutchinson, J. W. (1993) The influence of plasticity on mixed mode interface toughness. J. Mech. Phys. Solids 41, 1119-1135.

Tvergaard, V. and Hutchinson, J. W. (1994) Toughness of an interface along a thin ductile layer joining elastic solids. Phil. Mag. A 70, 641-656.

Varias, A. G., Suo, Z. and Shih, C. F. (1991) Ductile failure of a constrained metal foil. $J$. Mech. Phys. Solids 39, 963-986. 